\title{
2q13 microdeletion syndrome: Report on a newborn with additional features expanding the phenotype
}

\author{
Ettore Piro | Gregorio Serra (i) | Mario Giuffrè | Ingrid Anne Mandy Schierz | \\ Giovanni Corsello
}

Department of Health Promotion, Mother and Child Care, Internal Medicine and Medical Specialties "G. D'Alessandro", University of Palermo, Palermo, Italy

\section{Correspondence}

Gregorio Serra, Department of Health Promotion, Mother and Child Care, Internal Medicine and Medical Specialties "G. D'Alessandro", University of Palermo, Palermo, Italy.

Email: gregorioserra1984@libero.it

\begin{abstract}
We describe an additional newborn with craniofacial dysmorphisms, congenital heart disease, hypotonia, and a 2q13 deletion of $1.7 \mathrm{Mb}$. The clinical and genomic findings observed are consistent with the diagnosis of $2 \mathrm{q} 13$ microdeletion syndrome.
\end{abstract}

\section{K E Y W O R D S}

chromosome 2, CNVs, follow-up, genotype-phenotype correlations, newborn

\section{1 | INTRODUCTION}

In recent years, chromosomal microarray analysis (CMA) allowed the identification of a growing number of chromosomal imbalances within the human genome. ${ }^{1}$ The $2 \mathrm{q} 13$ region contains clusters of highly homologous sequences, which facilitate the occurrence of deletions and/or duplications. A recurrent $1.71 \mathrm{Mb}$ deletion is associated with a congenital syndrome (around 50 patients reported to date) manifesting with a variable phenotype (psychomotor development disabilities, mild craniofacial dysmorphisms, and congenital heart disease), and currently defined as $2 \mathrm{q} 13$ deletion or microdeletion syndrome. Hereby, we describe an additional newborn with craniofacial dysmorphisms, congenital heart disease, hypotonia, and a 2q13 deletion of $1.7 \mathrm{Mb}$. The clinical and genomic findings observed are consistent with the diagnosis of $2 \mathrm{q} 13$ microdeletion syndrome. This new neonatal case report provides additional genotypic and clinical data, as well as further insights into the possible biological and molecular bases of the 2q13 microdeletion syndrome.

These copy number variations (CNVs, defined as a greater than $1 \mathrm{~kb}$ segment of DNA that has variable copy number as compared to normal reference genome) include deletions and duplications ${ }^{2}$ and involve single or multiple genes. ${ }^{1}$ CNVs may be either benign or associated with diseases. ${ }^{2}$ Some regions of the genome are flanked by highly homologous sequences (segmental duplications, SDs, also known as low copy repeats). ${ }^{3}$ They are prone to mismatching and, then, to recurrent $\mathrm{CNVs}$, some of which cause clinically recognizable phenotypes (ie, Angelman, Prader-Willi, Smith-Magenis, and Williams-Beuren syndromes). ${ }^{1,4}$ The 2 q13 region contains clusters of such SDs, which facilitate recurrent deletions and/or duplications. These imbalances are less described than those affecting other chromosomes. ${ }^{4}$ A recurrent pathogenic $1.71 \mathrm{Mb}$ microdeletion is reported to date in around 50 patients. ${ }^{1}$ Their clinical phenotype is variable for the occurrence of mild (in the majority of cases) dysmorphic features, macro- or microcephaly, neurodevelopmental delay/behavior disorder (including hypotonia, impairment in fine and global motor skills, autism spectrum disorder, attention deficit, and hyperactivity disorder), as well as congenital heart disease (CHD, the most frequent anomaly), and other congenital defects (ie, scoliosis, renal/ genital malformations). ${ }^{5}$ 
Hereby, we describe an additional newborn with craniofacial dysmorphisms, CHD, hypotonia, and a 2q13 deletion of $1.7 \mathrm{Mb}$, whose diagnosis is consistent with $2 \mathrm{q} 13$ microdeletion syndrome. This new neonatal case report may provide additional genotypic and clinical data, as well as further insights into the possible biological and molecular bases of the 2q13 microdeletion syndrome.

\section{2 | PATIENT PRESENTATION}

This newborn female was the first and only born by vaginal delivery, from healthy nonconsanguineous parents, at $40^{+2}$ weeks of gestation after a naturally obtained and uneventful pregnancy. In the 26-year-old mother and 25-year-old father, any disease, as well as history of use of drugs and/or exposition to radiation, also before conception, was ruled out. At birth, her weight was $3200 \mathrm{~g}$ (39th percentile), length $50 \mathrm{~cm}$ (55th percentile), and occipitofrontal circumference (OFC) $34.5 \mathrm{~cm}$ (64th percentile). She was transferred on the second day of life from a first level birth center of the town, because of feeding difficulties, hypotonia, and dysmorphic features. On admission to our department, she showed on physical examination: facial asymmetry for hypoplasia of the left side of the face, low hairline, broad nasal bridge, pear-shaped tip of the nose and short columella, and small chin (Figure 1A). Posteriorly rotated left ear with thick helix, homolateral preauricular tag, and pit (Figure 1B), as well as inferotemporal epibulbar dermoid of the left eye and absent uvula, were observed. A sacral dimple was also noted (Figure 2). Generalized hypotonia and feeding difficulties completed her clinical profile. Brain ultrasound (US) showed a corpus callosum narrowing, between the anterior portion of the body and the genu. Abdomen and spinal cord US examinations showed no abnormalities. US heart evaluation revealed a subaortic ventricular septal defect. A whole spine X-ray was also performed and found lumbar kyphosis without vertebral defects.

Array comparative genomic hybridization (a-CGH) analysis (100-150 Kb resolution, genomic assembly GRCh37. p13) identified a $2 q 13$ deletion of $1.7 \mathrm{Mb}$ and indicated the positions 111,399,243 and 113,098,686 as the breakpoints of the rearrangement. The deleted region involved different genes, including partially BUB1, ACOXL, BCL2L11, MIR4435-2HG, MIR4771-2, ANAPC1, MERTK, TMEM87B, $F B L N 7, Z C 3 H 8$, and ZC3H6. a-CGH was also performed in both parents, showing normal results and, thus, confirming the de novo origin of the genomic abnormality.

The patient recovered from her initial feeding difficulties around the seventh day of life. Hearing screening through transient-evoked otoacoustic emissions (TEOAEs) revealed abnormal results. In order to ascertain and characterize the hearing loss, an audiological assessment was started. It included to date auditory brainstem response (ABR), which showed a left unilateral moderately severe conductive hypoacusis. She was discharged at 1 month of age after an otherwise uneventful clinical course and is included in a multidisciplinary (neurodevelopmental, surgical, audiologic, ophthalmologic, cardiologic) follow-up. She actually (3 months old) shows a mild developmental delay.

\section{3 | DISCUSSION}

The cytogenetic band $2 \mathrm{q} 13$ is enriched in clusters of highly homologous sequences, which facilitate the occurrence of deletions and/or duplications, quite recurrent in size and breakpoints. ${ }^{4}$ Among these rearrangements, a recurrent $1.71 \mathrm{Mb}$ deletion is associated with a congenital syndrome (around 50 patients reported to date) manifesting with variable phenotype, which includes psychomotor development disabilities, mild craniofacial dysmorphisms, and CHD, and currently defined as $2 \mathrm{q} 13$ deletion or microdeletion syndrome. ${ }^{1,5}$

The phenotype of the present patient (showing craniofacial dysmorphisms, CHD, and neurodevelopmental delay), although being overlapping with that of most $2 \mathrm{q} 13$ deletion subjects described to date, seems to be more severe (specifically for the eye and ear involvement), sharing clinical signs with the oculo-auriculo-vertebral spectrum (OAVS). This latter disorder affects craniofacial morphogenesis and

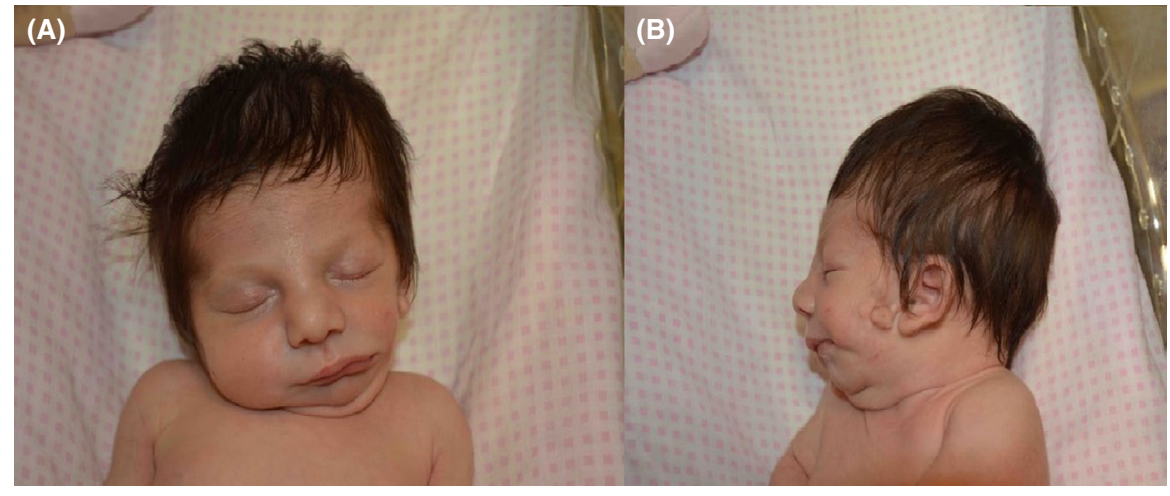

F I G URE 1 A, Note facial asymmetry for hypoplasia of the left side of the face, low hairline, broad nasal bridge, pear shaped tip of the nose, short columella, and small chin; B, the left ear is posteriorly rotated, with thick helix and preauricular tag and pit 


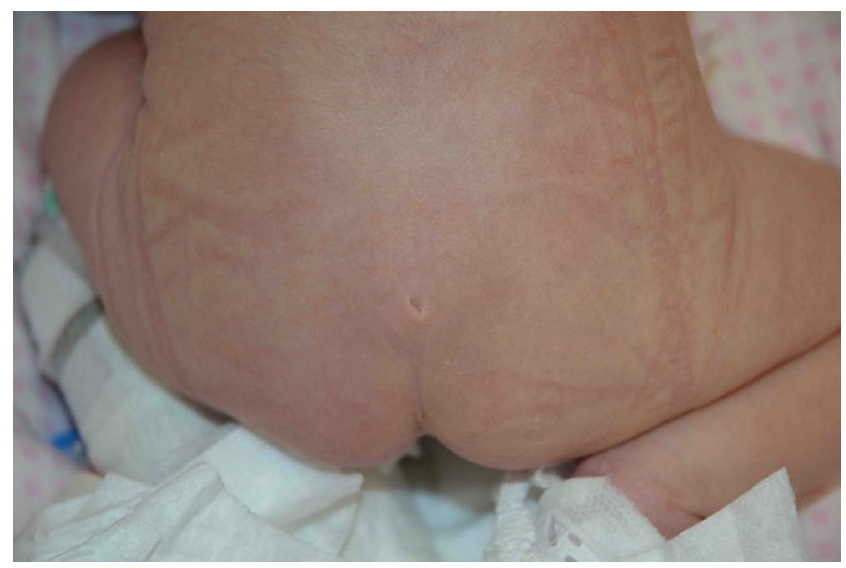

FIGURE 2 Sacral dimple

involves structures derived from the first and second pharyngeal arches. ${ }^{6}$ Its manifestations include asymmetric ear anomalies with or without hearing loss, hemifacial microsomia resulting in facial asymmetry, orofacial clefts, ocular (including epibulbar dermoids), and vertebral defects. Cardiac, genitourinary, and cerebral malformations may be associated. Several chromosomal/genomic anomalies are observed in patients showing an OAVS phenotype. However, 2q13 deletions are not reported. ${ }^{7}$

The deleted region of our patient encompasses eleven genes, nine protein coding ones $(B U B 1$ partially deleted, ACOXL1, BCL211, MERTK, FBLN7, TMEM87B, ANAPC1, $\mathrm{ZC} 3 \mathrm{H} 8$, and $\mathrm{ZC} 3 \mathrm{H} 6$ ), and two coding for microRNA (miRNAs, MIR4435-2HG, and MIR4771-2).

The dysmorphism and head circumference anomaly generally observed in cases with this deletion may depend on FBLN7 (fibulin 7) haploinsufficiency. This gene encodes fibulin-7, a glycoprotein which plays a role in the formation of facial bones. ${ }^{5}$ Its involvement may explain the hemifacial hypoplasia of our newborn, showing also absent uvula and small chin, as well as homolateral eye and ear involvement. Fibulin-7 cooperates with other proteins involved in heart development. Such function may clarify the association with CHDs reported in $2 \mathrm{q} 13$ deletion patients ${ }^{8}$ and observed also in our newborn. TMEM87B (transmembrane protein $87 \mathrm{~B}$ ) is likely related to developmental delays, autism spectrum disorders (ASDs), and other psychiatric phenotypes as well as $\mathrm{CHD},{ }^{5,8,9}$ and its defect may be consistent with the neurological (generalized hypotonia and feeding difficulties) and cardiac findings of our patient. Disruption of ACOXL (acylCoA oxidase like, affecting fatty acid metabolism) ${ }^{1,2}$ and BCL2L11 (BCL2-like 11, which encodes an antiapoptotic protein expressed in the frontal cortex and in the cerebellum, thus increasing apoptosis and the correlated risk of ASDs) ${ }^{10}$ may contribute to neurodevelopmental and ASD phenotypes of these subjects. ${ }^{1,2}$ ANAPCl (anaphase promoting complex subunit 1), a neurodevelopmental facilitator mainly expressed in the brain and the lymphoid organs, and MERTK
(MER proto-oncogene, tyrosine kinase) are candidate genes for the psychosis phenotype of $2 \mathrm{q} 13 \mathrm{CNV}$ carriers. ${ }^{1}$ The latter is also associated with retinal defects and autosomal recessive retinitis pigmentosa. It may have, thus, a role in the ocular development, which is disrupted (epibulbar dermoid) in our patient.

In the rearranged DNA of our newborn, there are also two genes coding for miRNAs that may influence the expression of the phenotype. They are short (20-24 nt) noncoding RNAs that are involved in post-transcriptional regulation gene expression. Between them, the MIR4435-2HG gene is ubiquitously expressed in different tissues, mainly brain, heart, and lymphoid organs. In the latter ones are also highly expressed ZC3H8 and ZC3H6 (zinc finger CCCH-type containing 8/6). Defects in one of these genes may cause a greater predisposition to infections (including otitis) and contribute to the phenotype of our proband, as well as to that of $2 \mathrm{q} 13$ deletion patients, in which such risk is described. ${ }^{1}$

Several explanations for the variable expressivity ${ }^{11}$ and reduced penetrance observed in $2 \mathrm{q} 13$ deletion patients are supposed, such as differences in gene content due to variable deletion breakpoints. ${ }^{4}$ Specifically, a more severe clinical expressivity (like that showed by our newborn, including craniofacial dysmorphisms, ocular involvement with epibulbar dermoid, and vertebral lumbar kyphosis) occurs because of the combined loss of more candidate genes present in the critical rearrangement. ${ }^{8}$ However, localization and width of such recurrent deletion is quite overlapping among the patients described in the literature. Therefore, besides the possible role played by genes contiguous to the deleted region (as occur in contiguous gene syndromes), there could be other plausible causes: In the "two hit" hypothesis, the $2 \mathrm{q} 13$ deletion could represent a "first hit" (ie a risk factor) for a second one, represented by an additional genomic imbalance (deletions and/or duplications), observed in some of the patients described to date, ${ }^{4}$ but which is absent in our proband; otherwise, other genomic mutations may unmask recessive alleles.

In the present patient, as whole genome sequencing was not performed, it cannot be ruled out that another genetic variant was contributing (or modify) to her phenotype. Moreover, epigenetic factors may also exacerbate the expressivity of the clinical picture. ${ }^{5}$ Our report is the first description of an OAVS phenotype in a 2q13 microdeletion patient. We cannot establish to date whether such association is casual, or whether a genetic bond exists between them. In regard to this, it could be supposed that loss of $F B L N 7$, which is expressed in the branchial arches, ${ }^{8}$ may be associated with development anomalies of the structures derived from the first and second pharyngeal arches (which are affected, indeed, in the OAVS).

Moreover, a further research on FBLN7 gene networks (https://genemania.org/search/homo-sapiens/FBLN7/, access 05/07/2020) identified a physical interaction 
network involving 3 genes (EDEM2, ASPH, and CLNSIA), a co-expression network involving 17 genes (GLYATL2, IZUMO2, SMYD2, BMPER, AHDC1, PRELID2, NEIL1, MDGA1, TLL1, TTC6, CDH7, EMID1, G6PC2, SH3GL3, $A M B N, D O C 2 A$, and $B C L 7 A)$, and a shared protein domain network involving TLL1. Among the aforementioned genes, three are associated with clinical phenotypes overlapping with the 2q13 deletion syndrome: TLL1 (cardiac malformations), ADHCl (hypotonia, corpus callosum hypoplasia), and $A S P H$ (facial dysmorphism). No FBLN7 gene pathway has been described yet.

\section{4 | CONCLUSION}

This study may provide useful data about the clinical and genomic features of 2 q13 deletions and expand the phenotypes associated with this rearrangement, which does not have to date a recognizable set of specific manifestations. ${ }^{12}$ It strengthens the importance of microarray analysis in cases of congenital malformations and/or dysmorphic features without a clinical picture suggestive for known disease. $^{13-15}$

The present report shows how an early genetic diagnosis, even more if obtained in neonatal age, may be crucial for the proper management of these patients. This must include individualized follow-up, which may allow prevention of future comorbidities (eg, infections, hearing loss, orthopedic, and neuropsychiatric disorders), as well as genetic counseling for the family, including communication of recurrence risk for later pregnancies. ${ }^{16}$

Further studies are needed to widen the knowledge on the molecular bases of the disease. The awareness of the role played by each gene included within 2 q13 region (functional studies, identification of single gene mutations), ${ }^{17}$ and the identification of further affected subjects, will allow its more precise genomic and clinical characterization, ${ }^{18}$ suggesting new elements of the genotype-phenotype correlations. ${ }^{19}$

\section{CONFLICT OF INTEREST}

The authors declare that they have no competing interests.

\section{AUTHOR CONTRIBUTIONS}

EP: performed neurological and developmental assessment, revised the manuscript, and gave final approval of the version to be submitted. GS: collected and analyzed clinical data, and drafted the manuscript. MG: contributed to clinical management of the patient and interpretation of genetical data. MS: contributed to clinical management of the patient, and in drafting the manuscript. GC: conceptualized the study, performed genetical assessment, revised the manuscript, and gave final approval of the version to be submitted. All authors approved the final manuscript as submitted.

\section{ETHICAL APPROVAL}

Written informed consent was obtained from both parents at the time of admission of our patient. The study was approved by the Mother and Child Department of the University of Palermo (Palermo, Italy). All procedures performed in this study were in accordance with the ethical standards of the institutional and national research committee, and with the 1964 Helsinki declaration and its later amendments or comparable ethical standards.

\section{DATA AVAILABILITY STATEMENT}

The data that support the findings of this study are available from the corresponding author upon reasonable request.

\section{ORCID}

Gregorio Serra (D) https://orcid.org/0000-0002-2918-9826

\section{REFERENCES}

1. Wolfe K, McQuillin A, Alesi V, et al. Delineating the psychiatric and behavioral phenotype of recurrent 2q13 deletions and duplications. Am J Med Genet B Neuropsychiatr Genet. 2018;177(4):397-405.

2. Yu HE, Hawash K, Picker J, et al. A recurrent $1.71 \mathrm{Mb}$ genomic imbalance at 2q13 increases the risk of developmental delay and dysmorphism. Clin Genet. 2012;81(3):257-264.

3. Rudd MK, Keene J, Bunke B, et al. Segmental duplications mediate novel, clinically relevant chromosome rearrangements. Hum Mol Genet. 2009;18(16):2957-2962.

4. Hladilkova E, Barøy T, Fannemel M, et al. A recurrent deletion on chromosome $2 \mathrm{q} 13$ is associated with developmental delay and mild facial dysmorphisms. Mol Cytogenet. 2015;8:57.

5. Guivarch J, Chatel C, Mortreux J, Missirian C, Philip N, Poinso F. An atypical autistic phenotype associated with a 2 q13 microdeletion: a case report. J Med Case Rep. 2018;12(1):79.

6. Passos-Bueno MR, Ornelas CC, Fanganiello RD. Syndromes of the first and second pharyngeal arches: a review. Am J Med Genet A. 2009;149A(8):1853-1859.

7. Beleza-Meireles A, Clayton-Smith J, Saraiva JM, Tassabehji M. Oculo-auriculo-vertebral spectrum: a review of the literature and genetic update. J Med Genet. 2014;51(10):635-645.

8. Russell MW, Raeker MO, Geisler SB, et al. Functional analysis of candidate genes in 2q13 deletion syndrome implicates FBLN7 and TMEM87B deficiency in congenital heart defects and FBLN7 in craniofacial malformations. Hum Mol Genet. 2014;23(16):4272-4284.

9. Yu HC, Coughlin CR, Geiger EA, et al. Discovery of a potentially deleterious variant in TMEM87B in a patient with a hemizygous 2 q13 microdeletion suggests a recessive condition characterized by congenital heart disease and restrictive cardiomyopathy. Cold Spring Harb Mol Case Stud. 2016;2(3):a000844.

10. Sheikh AM, Li X, Wen G, Tauqeer Z, Brown WT, Malik M. Cathepsin $\mathrm{D}$ and apoptosis related proteins are elevated in the brain of autistic subjects. Neuroscience. 2010;165(2):363-370.

11. Girirajan S, Rosenfeld JA, Coe BP, et al. Phenotypic heterogeneity of genomic disorders and rare copy-number variants. $N$ Engl J Med. 2012;367(14):1321-1331.

12. Riley KN, Catalano LM, Bernat JA, et al. Recurrent deletions and duplications of chromosome 2q11.2 and 2q13 are associated with variable outcomes. Am J Med Genet A. 2015;167A(11):2664-2673. 
13. Pavone P, Corsello G, Marino SD, Ruggieri M, Falsaperla R. 7q31.32 partial duplication: First report of a child with dysmorphism, autistic spectrum disorder, moderate intellectual disability and epilepsy. Literature review. Epilepsy Res. 2019;158:106223.

14. Pavone P, Marino SD, Corsello G, et al. Cerebral white matter lesions and dysmorphisms: signs suggestive of 6p25 deletion syndrome-literature review. J Pediatr Genet. 2019;8(4):205-211.

15. Viaggi CD, Cavani S, Pierluigi M, et al. Characterization of a complex rearrangement involving chromosomes 1,4 and 8 by FISH and array-CGH. J Appl Genet. 2012;53(3):285-288.

16. Lefebvre M, Sanlaville D, Marle N, et al. Genetic counselling difficulties and ethical implications of incidental findings from arrayCGH: a 7-year national survey. Clin Genet. 2016;89(5):630-635.

17. Iyer J, Girirajan S. Gene discovery and functional assessment of rare copy-number variants in neurodevelopmental disorders. Brief Funct Genomics. 2015;14(5):315-328.
18. Corsello G, Antona V, Serra G, et al. Clinical and molecular characterization of 112 single-center patients with Neurofibromatosis type 1. Ital J Pediatr. 2018;44(1):45.

19. Serra G, Antona V, Corsello G, Zara F, Piro E, Falsaperla R. NF1 microdeletion syndrome: case report of two new patients. Ital $J$ Pediatr. 2019;45(1):138.

How to cite this article: Piro E, Serra G, Giuffrè M, Schierz IAM, Corsello G. 2q13 microdeletion syndrome: Report on a newborn with additional features expanding the phenotype. Clin Case Rep. 2021;9:e04289. https://doi.org/10.1002/ccr3.4289 